\title{
Low altitude energetic electron lifetimes after enhanced magnetic activity as deduced from SAC-C and DEMETER data
}

\author{
S. Benck ${ }^{1}$, L. Mazzino ${ }^{2}$, M. Cyamukungu ${ }^{1}$, J. Cabrera ${ }^{1}$, and V. Pierrard ${ }^{1,3}$ \\ ${ }^{1}$ Center for Space Radiations (CSR), Chemin du Cyclotron, 2, 1348 Louvain-la-Neuve, Belgium \\ ${ }^{2}$ University of Alberta, Department of Physics, 11322 - 89 Avenue, Edmonton Alberta, Canada \\ ${ }^{3}$ Belgian Institute for Space Aeronomy (BISA), Ringlaan-3-Avenue Circulaire, 1180 Brussels, Belgium \\ Received: 26 June 2009 - Revised: 24 February 2010 - Accepted: 9 March 2010 - Published: 22 March 2010
}

\begin{abstract}
When flux enhancements of energetic electrons are produced as a consequence of geomagnetic storm occurrence, they tend to vanish gradually when the magnetic activity calms down and the fluxes decay to quiet-time levels. We use SAC-C and DEMETER low altitude observations to estimate the energetic electron lifetimes $(E=0.16-1.4 \mathrm{MeV}$, $L=1.6-5, B=0.22-0.46 \mathrm{G}$ ) and compare the decay rates to those observed at high altitude. While crossing the radiation belts at high latitude, the SAC-C and DEMETER instruments sample particles with small equatorial pitch angles ( $\alpha_{\text {eq }}<18^{\circ}$ for $L>2.5$ ) whereas the comparison is done with other satellite data measured mainly in the equatorial plane (for $\alpha_{\mathrm{eq}}>75^{\circ}$ ). While in the inner belt and in the slot region no significant lifetime differences are observed from the data sets with different $\alpha_{\text {eq }}$, in the outer belt, for the least energetic electrons $(<500 \mathrm{keV})$, the lifetimes are up to $\sim 3$ times larger for the electrons with the equatorial pitch-angle close to the loss cone than for those mirroring near the equator. The difference decreases with increasing energy and vanishes for energies of about $1 \mathrm{MeV}$.
\end{abstract}

Keywords. Magnetospheric physics (Energetic particles, precipitating; Energetic particles, trapped)

\section{Introduction}

Statistical space radiation models based on averages of all measured values include variances that result from stormtime transients. Such models fade away the characteristics of physical processes that drive the dynamic of particle

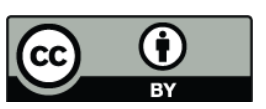

Correspondence to: S. Benck (benck@spaceradiatons.be) fluxes. We intend to break with classical model approaches and go beyond the existing static radiation models by introducing dynamic features based on observations of transient flux events. This approach is expected to facilitate the investigation of the physical mechanisms involved in the flux variations in the magnetosphere.

Quantifying the physical source and loss processes (Mc Ilwain, 1996; Friedel et al., 2002) controlling the electron dynamics in the radiation belts is the prerequisite for the development of a complete dynamic radiation belt model. While the quantitative determination of the source process (Obara et al., 2000; Meredith et al., 2002; O'Brien et al., 2003; Green and Kivelson, 2004; Onsager et al., 2004; Kataoka and Miyoshi, 2006; Hudson et al., 2008) parameters is outside the scope of this paper, a detailed description of the deduced parameter that will characterize the loss process in the model (i.e. the electron lifetime) will be given here. In fact, measurements of the lifetimes of energetic electrons are often considered to be the key to the understanding of the transport of these particles; for instance, they constitute key parameters in the determination of pitch angle diffusion rates (West et al., 1981).

Although the problem of particle loss by pitch angle diffusion driven by wave-particle interaction has been tackled up in the past (Lyons et al., 1972), intense interest currently still exists (Millan and Thorne, 2007) in quantification of these losses of energetic electrons in the radiation belts (Abel and Thorne, 1998a, b; Glauert and Horne, 2005; Shprits et al., 2006; Summers et al., 2007a, b; Meredith et al., 2007, 2009, and references therein). Nevertheless, most of these studies are based either on low altitude data acquired with limited energy resolution or on high altitude data. Our scope is to present electron lifetimes based on low altitude fluxes measured with high energy resolution, which may be used

Published by Copernicus Publications on behalf of the European Geosciences Union. 
to benchmark the theories under investigation. For this latter purpose, we revisit the determination of the L-shell dependence of electron timescales as a function of energy and position, and note a pitch-angle dependence of the observed lifetimes while comparing measurements from polar LEO (Low Earth Orbit), GTO (Geostationary Transfer Orbit) and HEO (Highly Elliptical Orbit) orbit.

The present study of electron lifetimes is based on satellite observations from SAC-C and DEMETER (Sect. 2). The decay timescales are deduced from a large flux data set covering more than five years of data, by applying a fit procedure to the decaying fluxes after high geomagnetic activity (Sect. 3). The results confirm a general trend in L- and E- dependence that has formerly been observed and qualitatively evaluated (Sect. 4) but where the absolute scale is still to be theoretically reproduced. The comparison of the decay rates of electrons observed at low altitude with the precipitation rates at the equatorial plane (Sect. 5), can give insight in the loss processes of high energetic electrons, dominated by wave-particle interaction for a large portion of the radiation belts $(L=1.6-5)$. Doing so, it is noticed that the average decay timescales at LEO significantly differ from those at the equatorial plane in the outer belt when the energy is below $500 \mathrm{keV}$. Discussion of possible explanations for this phenomenon is given in Sect. 6 .

\section{Instrumentation}

The SAC-C (Satélite de Aplicaciones Cientifico-C) international satellite was launched on 21 November 2000 into a sunsynchronous circular LEO orbit $\left(700 \mathrm{~km}\right.$ altitude, $98.2^{\circ}$ inclination, period $1 \mathrm{~h} 40$ ). Its primary mission is to provide multispectral imaging of terrestrial and coastal environments. Moreover, the spacecraft probes the structure and dynamics of the Earth's atmosphere, ionosphere and geomagnetic field. To measure the particle environment and its effects on electronics, it is equipped with the CNES (Centre National d'Etudes Spatiales, France) SPICA-ICARE (Spectre de Particules et Influence sur les Composants Avancés) instrument. The expected life time of SAC-C was two years, but it is still transmitting data.

ICARE (Influence of Space Radiation on Advanced Components) is composed of a set of three radiation detectors associated with a component test board (Falguère et al., 2002). It performs two types of measurements: the radiation environment characterization and its effects on components. The radiation detectors are made of fully depleted solid state detectors used in single and/or coincident and/or anti-coincident mode. These three units of the ICARE instrument provide electron, proton and ion $(\mathrm{He})$ fluxes in a wide range of energies. The first one (the "E" detection head) is made of a $500 \mu \mathrm{m} \mathrm{Si} \mathrm{diode} \mathrm{placed} \mathrm{in} \mathrm{a} 5 \mathrm{~mm}$ thick cylindrical aluminum shield. A window of aluminum $(50 \mu \mathrm{m}$ thick, $3 \mathrm{~mm}$ in diameter) gives free access to low energy elec- trons $(E>130 \mathrm{keV})$ and protons $(E>2.5 \mathrm{MeV})$ to the diode with a field of view angle of about $\pm 42^{\circ}$ (Bourdarie et al., 2006). Nevertheless, through electronic threshold settings, only fluxes of $E>190 \mathrm{keV}$ electrons and $E>3 \mathrm{MeV}$ protons can be measured. With the "E" head, only $E<1.2 \mathrm{MeV}$ electrons can be registered contamination free (Ecoffet et al., 2005). The second unit (the "P" detection head) is made of two Si junction detectors $(150 \mu \mathrm{m}$ and $6000 \mu \mathrm{m}$ thick), placed in a $5 \mathrm{~mm}$ thick cylindrical aluminum shielding (Outer diameter $25.5 \mathrm{~mm}$ ) protected by $500 \mu \mathrm{m}$ thick aluminum windows $(15.5 \mathrm{~mm}$ in diameter) on the front and rear side of the cylinder. Its main function is to measure proton spectra but also higher energy electron fluxes. The third unit ("I" detection head) is dedicated to ion detection. The omnidirectional differential electron fluxes (retrieved from http: //www-mip.onera.fr/rermm/SACC/) are given within the energy range $0.2-4.11 \mathrm{MeV}$. The electron fluxes used within this study range from 0.23 to $1.36 \mathrm{MeV}$ (10 energy bins of variable size: $0.23-0.29,0.29-0.35,0.33-0.39,0.35$ $0.45,0.45-0.51,0.53-0.59,0.59-0.65,0.64-0.76,0.76-$ $0.88,1.08-1.36 \mathrm{MeV}$ ) and are registered during the time period December 2000 to September 2006.

The DEMETER (Detection of Electro-Magnetic Emissions Transmitted from Earthquake Regions) satellite was launched on 29 June 2004 into a circular polar sunsynchronous orbit at an altitude of $710 \mathrm{~km}$ with an inclination of $98^{\circ}$ (Parrot, 2006, http://demeter.cnrs-orleans.fr/). The orbital period is $\sim 1 \mathrm{~h} 40$. The scientific payload is switched off over the poles and higher latitude auroral regions.

The particle data used in this study are provided by the Instrument for Particle Detection (IDP) devoted to the measurement of electron fluxes along the DEMETER orbit. The instrument (Sauvaud et al., 2006) consists of a $1 \mathrm{~mm}$ thick silicon detector behind a collimator that defines the field of view of $\pm 16^{\circ}$. The optic has also an aluminum foil with a thickness of $6 \mu \mathrm{m}$ to avoid parasitic light and to stop protons with energies lower than $\sim 500 \mathrm{keV}$. The detector looks perpendicularly to the orbital plane of the satellite so that the pitch angle of the detected particles is close to $90^{\circ}$. Flux measurement is achieved by counting particles that deposit energy in the $70-2340 \mathrm{keV}$ range in the detector. The instrument has been fully characterized in-beam and by a GEANT-based Monte Carlo simulation. It was found to efficiently detect electrons in the $70 \mathrm{keV}$ to $\sim 1.2 \mathrm{MeV}$ energy range with limited contamination from protons. To achieve differential flux measurement, this $70-2340 \mathrm{keV}$ energy interval is divided into 256 channels when operating in the so-called "BURST mode". In "SURVEY mode", the IDP data are stored in 128 channels. For convenience, we grouped the energy channels in order to consistently work with 27 channels independent of the operating mode. Each new channel covers an energy range of about $90 \mathrm{keV}$. Spectra are given with a time resolution of 1 or $4 \mathrm{~s}$ depending on the operational mode (Burst or Survey). Also, it must be mentioned that the 14 first channels out of the 27 show an efficiency to electrons high enough 
to allow electron detection, the following channels may be a mixture of electrons and protons and the last channels detect more or less exclusively protons. Within this study the electron energy channels used, range from $162 \mathrm{keV}$ to $1.15 \mathrm{MeV}$ (11 channels of about $90 \mathrm{keV}$ : $0.16-0.26,0.25-0.34,0.34$ $0.43,0.43-0.52,0.52-0.61,0.61-0.70,0.70-0.79,0.79-$ $0.88,0.88-0.97,0.97-1.06,1.06-1.15 \mathrm{MeV})$ and the covered time period goes from August 2004 to March 2006.

Due to their low earth orbits, the satellites sweep through the radiation belts only at high latitude or in the South Atlantic anomaly (SAA). The McIlwain $(B, L)$ coordinate system is used to represent the data. A local version of the UNILIB library (www.oma.be/NEEDLE/unilib.php) is used to calculate the positions in the $(\mathrm{B}, \mathrm{L})$ coordinate system from the geographic positions given by the satellite data provider. The field strength $B$ is calculated using internal and external field models: The internal magnetic field component is calculated using the IGRF2000 coefficients extrapolated to the date of measurement. To calculate the external field component the Tsyganenko 1989 model was selected with Kp set to $0^{+}$. The $\mathrm{L}$ parameter was determined using a magnetic dipole moment of $M=0.311653 \mathrm{GRe}^{3}$. The analysis was restricted to data in the L-region 1.6-5 and $\mathrm{B}$ region $0.22-0.46 \mathrm{G}$. If we assume a dipole field and that the local pitch angle of the detected particles is $\leq 90^{\circ}$ (close to $90^{\circ}$ for Demeter data and variable for SAC-C) then these assumptions restrict the analysis to equatorial pitch angles $\alpha_{\mathrm{eq}}$ in the range $\alpha_{\mathrm{eq}}<25^{\circ}$ at $L=2$ and $\alpha_{\mathrm{eq}}<6^{\circ}$ at $L=5$.

\section{Data analysis: determination of the decay timescales}

The method to automatically determine the electron lifetimes is similar to that adopted in the work of Meredith et al. (2006). The constraints on the method were however adapted to comply with the characteristics of the data and are described here below. The fluxes are assumed to decay exponentially to the quiet time level. Therefore the decay timescales, $\tau$, were obtained by fitting a linear function to the natural logarithm of the fluxes (least square fit). The strength of the linear relationship is given by the correlation coefficient $r$ that varies between -1 and 1 . The correlation coefficient is determined for the 12 first points in the dataset $(=12$ days for a time resolution of 1 day for $L>2.4$ ). If $r$ is negative with an absolute value less than 0.94 or positive, the fit is classified as "not good" and the starting point is incremented by one point and the next set of 12 points is fitted. If $r$ is negative and its absolute value is greater than 0.94 , the number of points included in the fit is increased by one unit until the value of $r$ becomes less than 0.5 in absolute. The fitting interval is then chosen to be the fit with the highest absolute value of $r$, which is in that case greater than or equal to 0.94 . The increment in the number of data points for the fit and the selection of the fit with the best correlation coefficient, imply that the retained fit is based on at least 12 points and has a correlation coefficient better than 0.94. The time span over which a decay time constant is deduced may therefore vary. To use just good quality data and avoid regions of big data dispersion, the fitting curve must all the time be within $25 \%$ of the data. However, the fitting technique breaks down when $L$ is lower than 2.6. For these cases, the limit in $r$ (standard 0.94 ) and the number of minimum points (standard 12) was adapted so as to obtain reasonable good fits. For data with $L<2.4$, a 2 days time resolution was used, as the lifetimes start to increase significantly when entering the inner belt.

The SAC-C/ICARE differential electron fluxes of 0.23$0.29 \mathrm{MeV}$ electrons at $L=2.1,2.9$ and 4.1 (center value of the bin, $\Delta L=0.2$ ) and the Dst index are plotted as a function of time from 1 March 2001 to 1 November 2001 in Fig. 1. The restriction in the magnetic field range $B=0.22-0.46 \mathrm{G}$ has been imposed to avoid data from the South Atlantic Anomaly in the heart of which the instrument may be affected by saturation. Large increases in the flux of energetic electrons are associated with increased magnetic activity as monitored by the Dst index (the orange lines identify geomagnetic storms with a Dst minimum larger than $50 \mathrm{nT}$ in absolute). The flux enhancement can be very rapid and may take place over a timescale of about half a day to four days depending on the magnetic and wave activity as well as on the enhanced fluxes of the seed population. Fewer events were recorded at the lowest L-shells as only the strongest geomagnetic storms affect this region. The electron decay timescales in that region are also very large. While close to the slot region the fluxes are only affected by stronger storms, the particle fluxes in the outer belt react already to much weaker geomagnetic activity variations (cf. time period July-September 2001 on Fig. 1). The fits fulfilling the requirements described above are over plotted on the data in Fig. 1 (blue curves) and the respective lifetimes are indicated. The lifetimes show a certain dispersion depending on the after storm conditions. For instance, from a diffusion analysis, West et al. (1981) found evidence to support the assumption that the most rapid decays are those observed when radial diffusion is weak and pitch angle diffusion is identified as the predominant process responsible for the decay.

\section{Results}

The data analysis was repeated for L-values between $L=1.4-$ 5.0 in steps of 0.2 for all the energy channels of the SAC$\mathrm{C}$ and DEMETER particle instruments between 0.16 and 1.4 MeV. The results from both sets of data were put together: the lifetime data from overlapping energy bins are averaged and the extreme energy limits were taken to define the new energy bin within which the mean lifetime value is valid. Figure 2 shows an example of lifetimes obtained from the two sources of flux data, i.e. SAC-C (black diamonds) and DEMETER (red triangles) satellites, as a function of time of measurement. The mean lifetime value quoted on the graph 

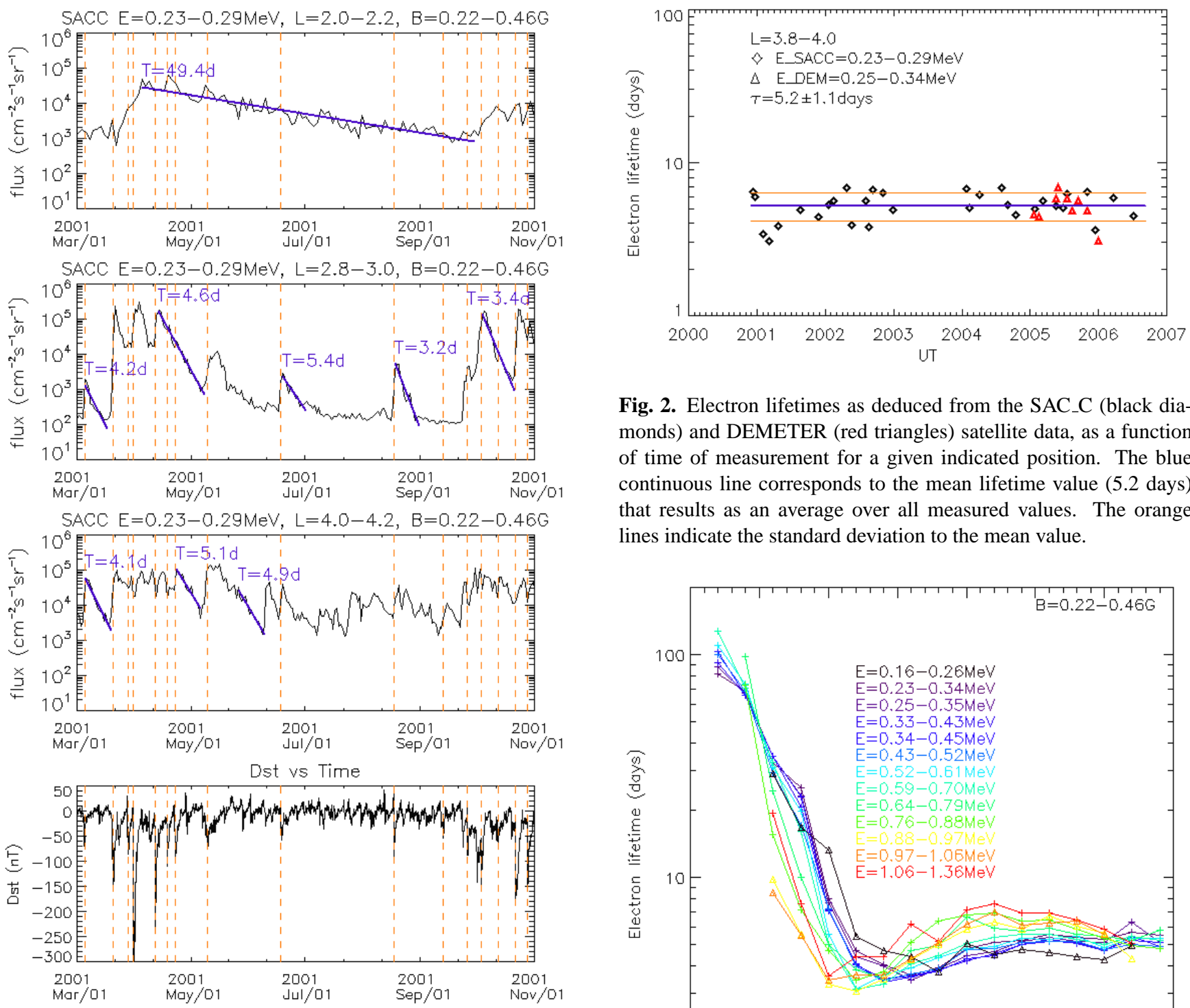

Fig. 2. Electron lifetimes as deduced from the SAC_C (black diamonds) and DEMETER (red triangles) satellite data, as a function of time of measurement for a given indicated position. The blue continuous line corresponds to the mean lifetime value (5.2 days) that results as an average over all measured values. The orange lines indicate the standard deviation to the mean value.

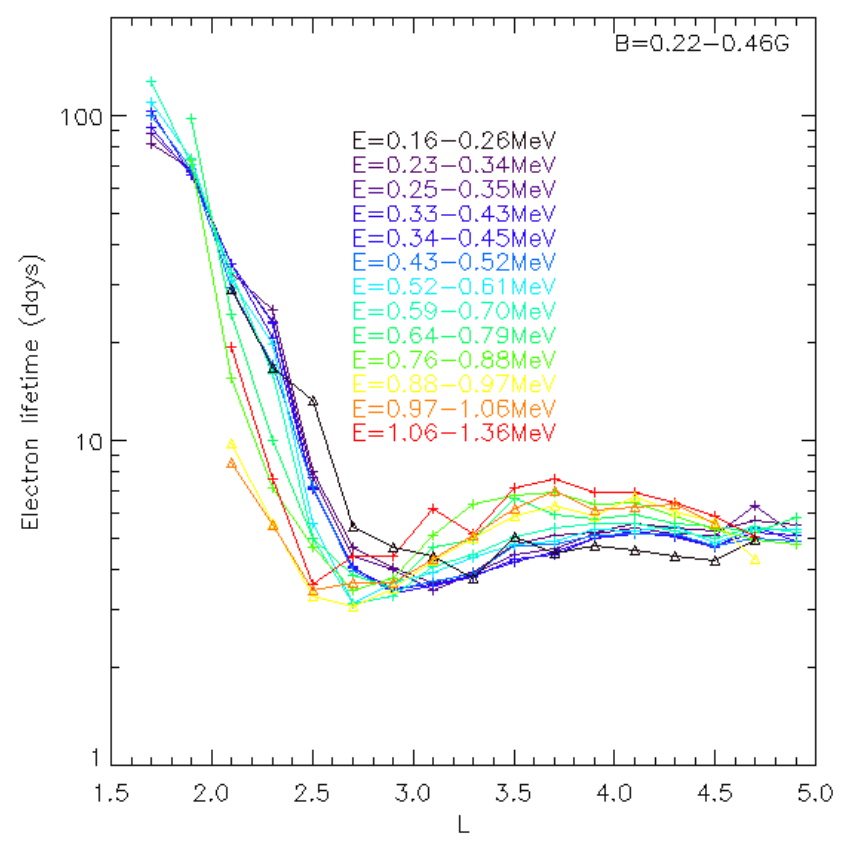

Fig. 1. The three upper panels show the SAC-C/ICARE differential electron fluxes of $0.23-0.29 \mathrm{MeV}$ electrons at the indicated positions (given by $L$ and $B$ ) as a function of time from 1 March 2001 to 1 November 2001. The last panel gives the Dst index for the same time period. The orange lines indicate the instant of Dst minimum (for $-\mathrm{Dst}_{\min }>50 \mathrm{nT}$ ). The blue curves indicate the result of the fitting procedure. The corresponding electron lifetimes are also indicated.

(shown as the blue continuous line in Fig. 2 is an average over all measured values. The orange lines indicate the standard deviation to the mean value. It can be observed that the average lifetime stays constant during the long time period from solar maximum in 2001 through the declining phase until 2006.

The experimental electron lifetimes $\tau$ as a function of $L$ and $E$, as well as the number of events $n$ used in the determination of these mean lifetimes are given in Table 1 . The

Fig. 3. Measured mean electron decay timescales versus $L$ for the indicated energy ranges.

quoted errors are the standard deviations on the mean value. The typical uncertainty (relative error) on the lifetime values is $30 \%$. When the decay timescale is only defined by one value then the error bar is based on this typical uncertainty. 


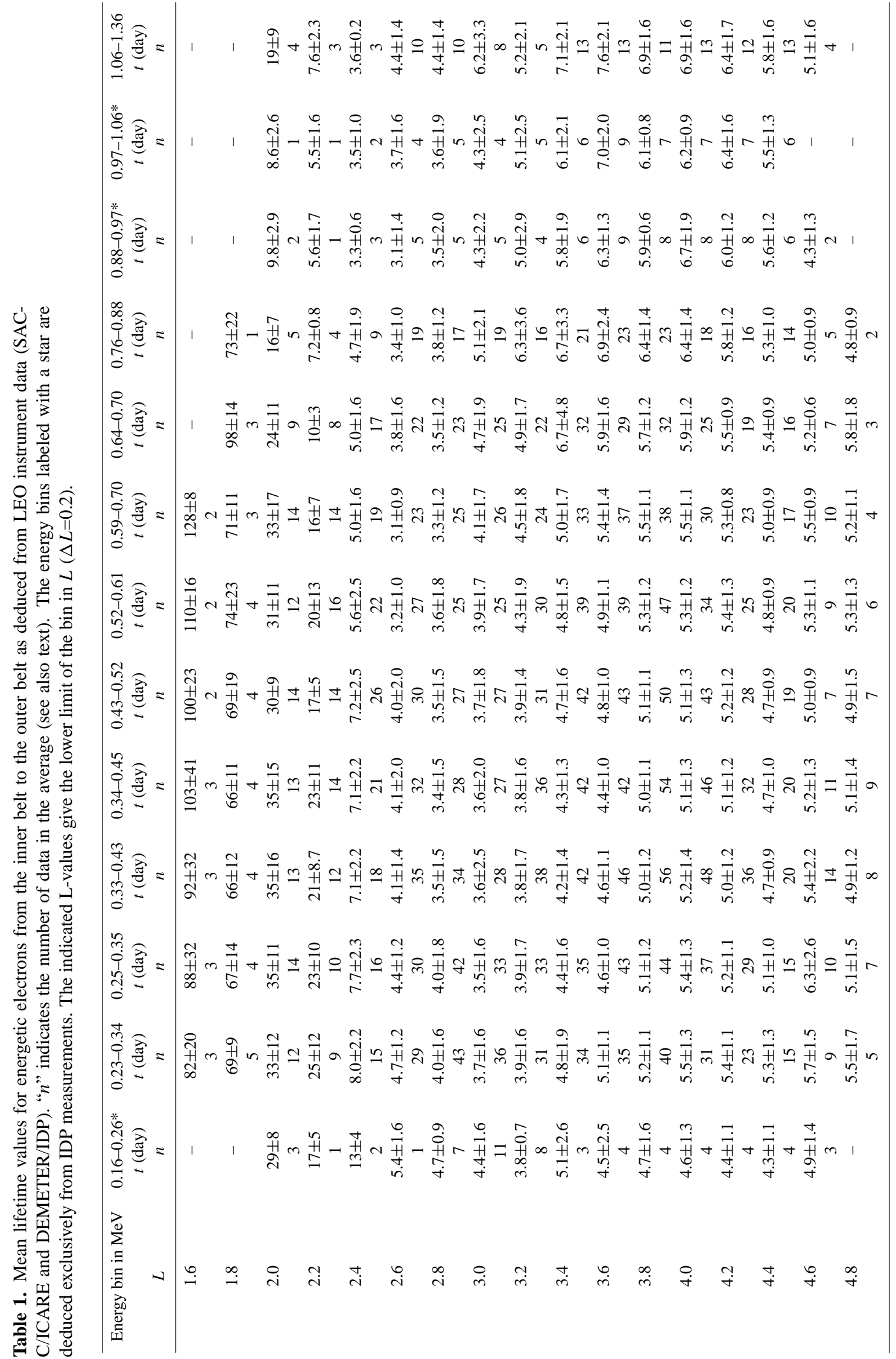


The observed decay timescales are plotted, for the resulting energy bins, as a function of $L$ for the region $1.6 \leq L<5$ in Fig. 3. The inner belt regions are characterized by very high decay time constants that increase quickly with decreasing $L$. The largest observed lifetimes in this case are larger than 3 months. Looper et al. (1994) also observed that at the outer edge of the inner belt electron lifetimes can be very large and reach up to 6-12 months for relativistic electrons $(E \geq 15 \mathrm{MeV})$. In the inner belt, the general trend is that the highest decay times are observed for the less energetic particles. This observation has also been noted in former investigations for relativistic electrons in the heart of the inner radiation belt (Freden, 1969). In the slot region, for all the energy bins considered, the electron decay timescales have a minimum of 3-4 days near $L=2.5-3.2$ (energy dependent). It has been observed that the location of the minimum lifetime for a given energy depends on the after storm conditions. In the outer belt, the lifetimes slightly increase with increasing $L$, but the electrons with the highest energy decay the slowest (largest decay time constants). This is a trend that is generally observed after individual storms (Benck et al., 2008) but that is not exclusive. Meredith et al. (2006) also showed a very clear energy dependence of the decay timescales for energetic electrons in the $0.2-1.1 \mathrm{MeV}$ range for outer belt electrons $(3<L<5)$.

\section{Comparison with existing data}

The SAC-C/ICARE and DEMETER/IDP particle detectors measure electrons which are stably trapped and mirror at low altitudes (dominant component) together with precipitating electrons in the loss cone. Assuming a dipole magnetic field, a $90^{\circ}$ pitch angle measured for ICARE or IDP at $L=2$ corresponds to an equatorial pitch angle of approximately $18^{\circ}$. Electrons with pitch angle greater than this mirror before reaching the satellites. The measured lifetimes are therefore restricted to the decay of the equatorial electron distribution close to the loss cone. To determine whether the decay timescales of electrons are pitch angle dependent, the LEO data are compared to existing experimental lifetimes based on electron data measured closer to the magnetic equator. Table 2 gives a summary of the characteristics of the satellites and the flux data on which the other lifetime results are based. For direct comparison with our measurements we only considered data sets that are based on fluxes given for well defined energy bins i.e. lifetime data based on integral fluxes $\phi\left(E>E_{\text {threshold }}\right)$ measurements were discarded. The data from the study described in Vampola et al. (1971) were taken from the graph in Lyons et al. (1972). Based on the remarks on the OV3-3 data given by West et al. (1981), it was deduced that the lifetimes of Vampola et al. (1971) were partly deduced from fluxes with pitch angle as low as $20^{\circ}$ ( $B=0.05 \mathrm{G}, L=3.5$ ).
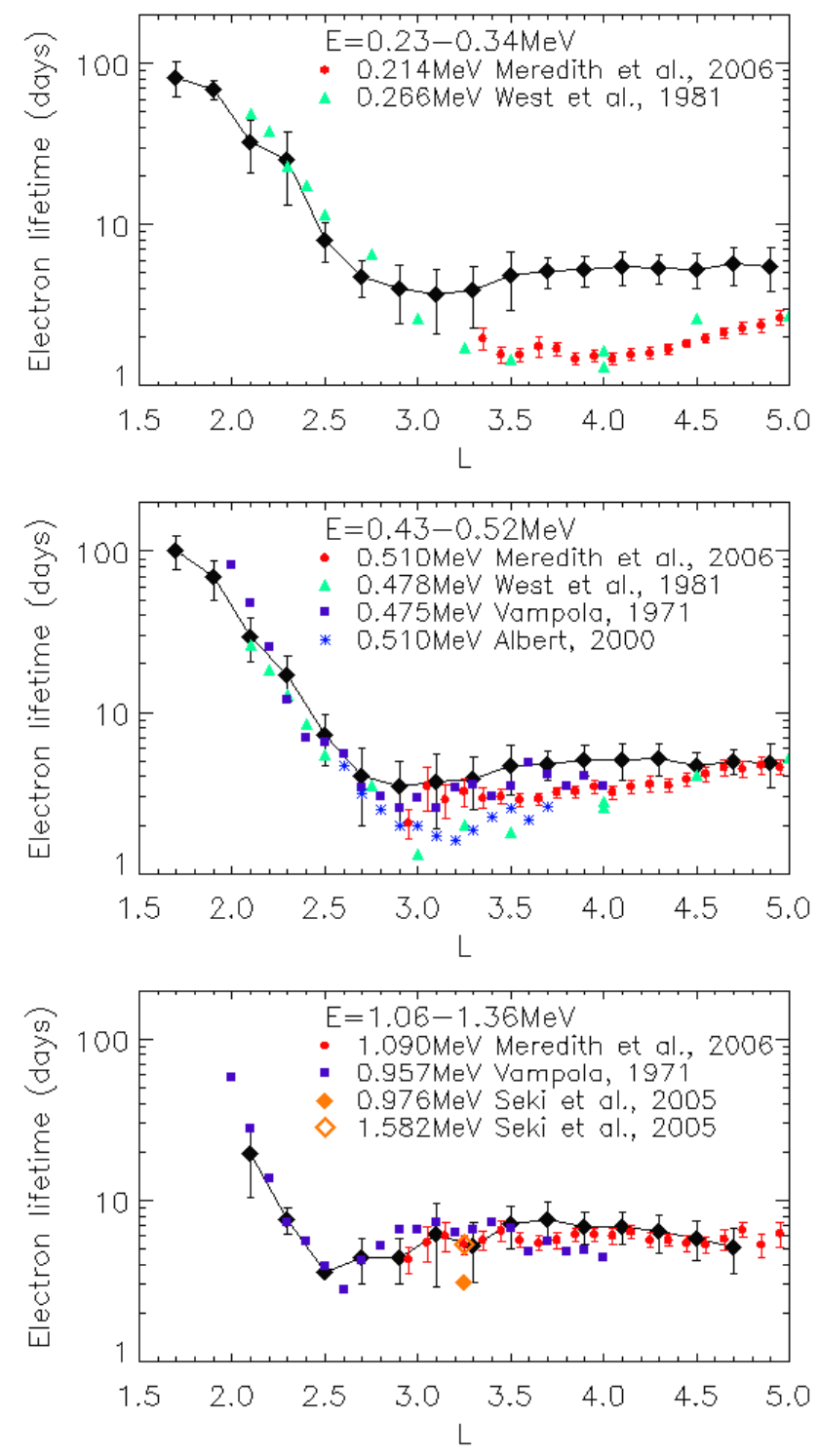

Fig. 4. Observed lifetimes (black dots) versus $L$ for three different energy ranges. Comparison to other measured decay timescales taken from the indicated references.

Figure 4 shows the decay times of electrons for three energy bins $(0.23-0.34 \mathrm{MeV}, 0.43-0.52 \mathrm{MeV}, 1.06-1.36 \mathrm{MeV})$ as a function of $L$ compared to the other experimental results. It can be observed that while in the inner belt and in the slot region there is no significant difference from the data sets with different $\alpha_{\mathrm{eq}}$, in the outer belt for the least energetic electrons $(<500 \mathrm{keV})$ the lifetimes are up to $\sim 3$ times larger for the low altitude electron fluxes than for their equatorial counterparts. The difference decreases with increasing energy and vanishes for energies about $1 \mathrm{MeV}$. For the $0.43-$ $0.52 \mathrm{MeV}$ energy bin, the data from Vampola et al. (1971) agree with our measurements. This is not surprising as these data are deduced from high latitude fluxes. Albert (2000) 
Table 2. Data sources (and their characteristics), from which lifetimes have been deduced. The last column gives indications on the flux specifics in the data analysis $\left(J_{\perp}\right.$ is the flux in the direction perpendicular to $B$ at the geomagnetic equator $\left(J_{\perp}=J\left(\alpha_{\mathrm{eq}}=90^{\circ}\right)\right)$.

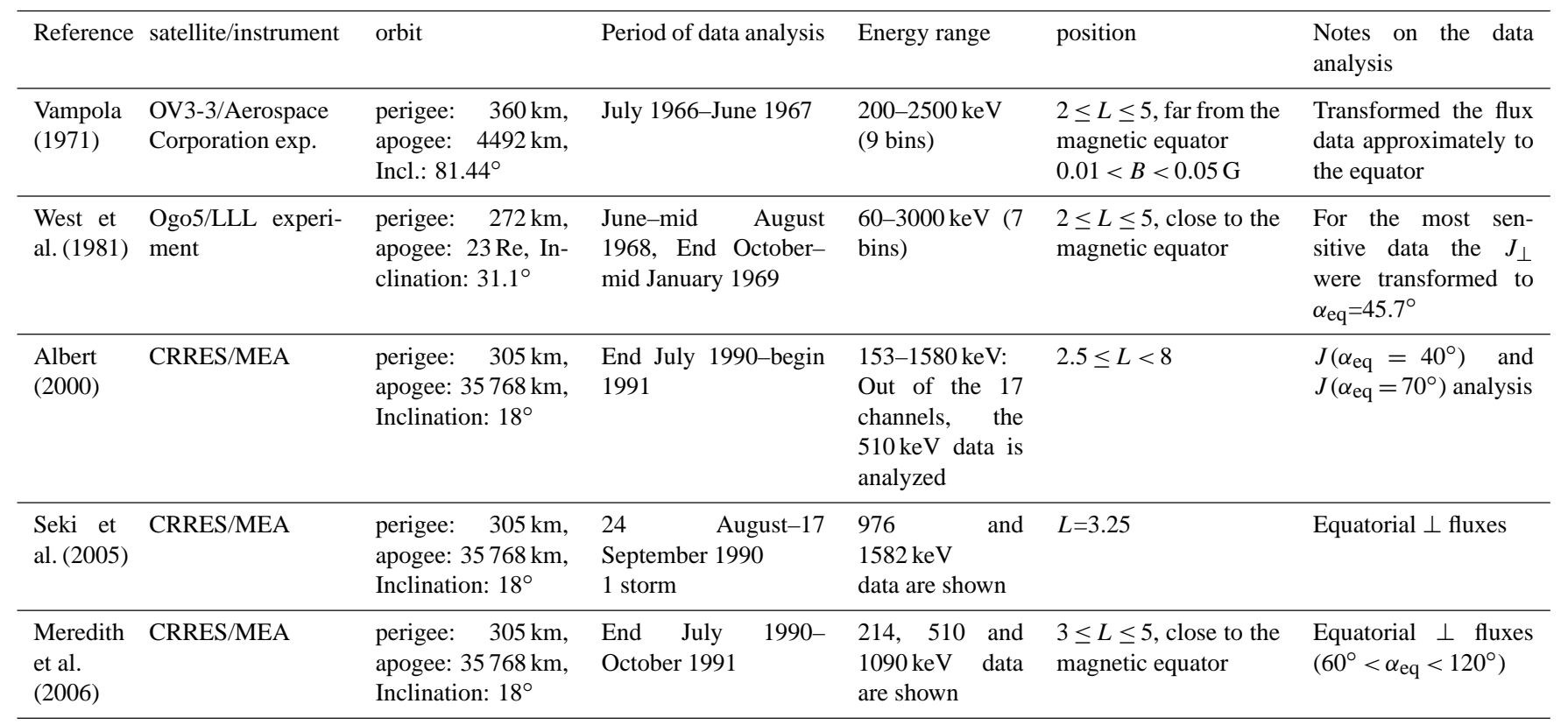

showed decay time constants for $510 \mathrm{keV}$ electrons, for two equatorial pitch angles $\left(\alpha_{\mathrm{eq}}=40^{\circ}\right.$ and $\left.70^{\circ}\right)$ and observed that at this energy there is no difference in the lifetimes from the two particle flux populations. However, the present study based on LEO fluxes $\left(\alpha_{\mathrm{eq}}<20^{\circ}, L>2.5\right)$ gives lifetime constants that are larger for $L>2.8$. The timescales from Meredith et al. (2006) who averaged CRRES/MEA lifetimes over a larger period, are somewhat larger than the values from Albert (2000), but still slightly below the results presented herein. When reaching the highest treated energy bin (1.06-1.36 MeV), all the data scatter close to one line.

Beyond the $\mathrm{L}$ and $\mathrm{E}$ domain covered by our analysis, several interesting features can be pointed out:

- Fig. 4, in the region of the inner belt (here $1.6 \leq L<$ 2.4), does not show any difference in deduced lifetimes coming from equatorial plane or low altitude measurements. This is not in contradiction with previous observations from Beall et al. (1967) who showed, that in the inner belt, there exists a dependence of the electron lifetime on $B$ (or $\alpha_{\mathrm{eq}}$ ) but that this dependence seems to vanish when moving to higher L-shells $(L>1.35)$.

- Baker et al. (2007) presented lifetimes for relativistic electrons of energy $2-6 \mathrm{MeV}$ for the region $1.5 \leq L \leq$ 2.5 deduced from SAMPEX observations. This analysis was later extended to $2 \leq L \leq 3$ and the results are analyzed in Meredith et al. (2009). They continue the general trend of our data: At the outer boarder of the inner belt $(2<L<2.5)$ the lifetimes for $2-6 \mathrm{MeV}$ electrons are generally lower than the lifetimes for the 1.061.36 MeV electrons. Moving towards the outer belt, the lifetimes for the 2-6 MeV electrons become larger than the lifetimes determined for the highest energy bin in the present study.

- Baker et al. (1994) analyzed one year of SAMPEX data and estimated decay timescales of 5 to 10 days for electrons with energies higher than $400 \mathrm{keV}$ in the region $2.5<L<5$. While a direct comparison with a specific energy channel of our study is not possible in this case, the range of lifetime values has some overlap with the range of values determined within our study for $E>430 \mathrm{keV}$ and $L>2.6$ (see Table 1). The overlap becomes considerable when comparing their lifetime range to our values for the last energy bin 1.061.36 MeV. Given that in the mentioned L-region, high energy electrons have a slower decay rate than low energy electrons, it can be understood that these data from low altitude measurements tend to be somewhat higher than our deduced lifetimes around $500 \mathrm{keV}$. McIlwain (1996) showed equatorial flux variations of electrons with energies above $500 \mathrm{keV}$ that exhibit a decay time constant as large as 16 days. In their comparison of electron flux characteristics at low and high altitude, Williams et al. (1968) observed no difference between lifetimes for electrons with energies above $\sim 280 \mathrm{keV}$ at both positions. The difference may have been faded 
away by the broad energy range. This points-out the importance of studying flux characteristics with enough resolution (energy, position, pitch-angle). By taking unlimited energy bins, the lifetime constants may be overestimated and interesting features may be shadowed.

\section{Discussion}

The outcome of this lifetime analysis is that for energies below $500 \mathrm{keV}$ the pitch-angle distribution does not decay at a single rate independent of equatorial pitch angle $\alpha_{\mathrm{eq}}$; this feature is in contradiction with what is generally assumed and theoretically predicted (Albert and Shprits, 2009) and needs to be investigated.

The lifetimes presented here are an average over all the values observed during several time intervals at low altitude. But, it is well known that there are many processes acting simultaneously upon the particle population within the magnetosphere i.e. pitch-angle diffusion due to wave-particle interaction or Coulomb collision, energy loss, radial transport, acceleration, injection. The relative importance of these processes during specific time intervals determines whether the fluxes are increasing or decreasing and at what rate this is occurring. Possible effects of the most relevant processes are discussed hereafter:

Pitch-angle diffusion is one of the major processes that determine the electron loss rates and their pitch-angle distributions during the recovery phase of a storm or during low geomagnetic activity. West et al. (1981) considered that the lowest lifetimes in the decays of the fluxes identify the pitchangle diffusion lifetimes. Applying the same reasoning, lifetimes of about 3 days would be deduced for $L=3.9$ and electron energy of $0.23-0.34 \mathrm{MeV}$ (see Fig. 2) if pitch-angle diffusion is the only process in action. This observed low altitude lifetime value is still approximately twice the value observed by Meredith et al. (2006). Here it must be stated that this latter reference also gives mean lifetimes values from data obtained over a 15 months period and that these data have not either been sorted for lowest values to obtain pure pitch angle diffusion lifetimes. Even so, assuming that the equatorial data are the result of pure pitch-angle diffusion, an explanation needs to be found why does the difference in lifetime persist or why does the pitch angle distribution not evolve to an equilibrium shape for $E<500 \mathrm{keV}$. Generally in that region, interaction with plasmaspheric hiss is the dominant mechanism responsible for the pitch-angle scattering (Abel and Thorne, 1998a). One process needs to be found that would enable the distribution to evolve less quickly to an equilibrium state. Meredith et al. (2006) stated that plasmaspheric hiss appear to propagate over a broad range of wave normal angles with predominantly field-aligned propagation near the geomagnetic equator and more oblique propagation at higher latitudes. Comparing their experimental lifetime data to theoretical results obtained for different wave nor- mal angles, they observed that plasmaspheric hiss propagating at small or intermediate wave normal angles are responsible for electron loss over a wide range of L-shells and energies. Plasmaspheric hiss with large wave normal angles do not contribute significantly to the electron loss. This goes in hand with the formerly stated fact that field-aligned propagation is dominant near the equator: the particles mirroring at the equator are permanently embedded in the wave field with small wave normal angles and therefore are subjected to lifetimes determined by these waves. It may now be that particles mirroring at higher latitudes that pass most of their time in regions where oblique wave propagation is present, tend to interact predominantly with these oblique waves and this as a function of decreasing energy. Based on the theoretical results shown in Fig. 10 of Meredith et al. (2006), we estimated that if at $L=4$ and $E=214 \mathrm{keV}, 40 \%$ of the electron population interacted with the hiss having a wave normal angle of $80^{\circ}$, the lifetime for that energy would be close to 5 days. For $L=4$ and $E=510 \mathrm{keV}$, a lifetime of 5 days can be reached if about $15 \%$ of the electron population interacts with the highly oblique waves. At low $L$, in the slot region and inner belt, other wave emissions such as lightening generated whistlers and VLF transmitters, become increasingly important and contribute to the decay timescales in that regions (Abel and Thorne, 1998a). Therefore at low $L$, the characteristics of the plasmaspheric hiss - electron interaction are less dominant for particle scattering and these additional waves may equilibrate the decay rate of $<500 \mathrm{keV}$ electrons to a single rate, independent of equatorial pitch angle.

The other processes that act upon the particle population and that possibly contribute to the decay rate of electrons of $E<500 \mathrm{keV}$ and $\alpha_{\mathrm{eq}}<20^{\circ}$ in the outer zone also need to be investigated. Inside the high density plasmasphere, energy diffusion as a result of gyroresonant wave-particle interactions is small compared to pitch angle diffusion. Furthermore, the timescale for pitch angle scattering loss of $\mathrm{MeV}$ electrons in the slot region is typically orders of magnitude shorter than that for radial diffusion (Meredith et al., 2009), i.e. the time evolution of the $\mathrm{MeV}$ electron distribution is dominated by the pitch angle diffusion process. The situation may however be different when getting closer to the plasmapause at $L \sim 4$ : the observed decay rates of electrons of a given energy and equatorial pitch angle may be modified by the effects of energy diffusion or radial diffusion transport. Brautigam and Albert (2000) observed that lower energy particles are subject to inward radial diffusion while higher energy electrons diffuse outward. If inward radial diffusion could have a significant effect on the loss, then the decay timescales of lower energy electrons would be higher than those of $\mathrm{MeV}$ electrons as it is typically more effective on lower-energy particles (Gannon et al., 2007). Moreover, inward radial diffusion causes a flux increase around $\alpha_{\mathrm{eq}}=90^{\circ}$ and so the global loss rate of the particle population mirroring at the equator should be slowed down and the lifetime 
of equatorially mirroring particles should be higher than that of particles close to the loss cone. This is however not observed, which suggests that inward radial diffusion cannot be responsible for the observed phenomena

Another mechanism that may explain the lower observed decay rates for non-equatorially mirroring particles is acceleration. Wave acceleration is most efficient in low-density regions, therefore this process should take place outside the plasmasphere within which hiss dominate (Horne et al., 2005). At $L=4$ we are at the border of the plasmapause and this criteria may be partially fulfilled. Horne at al. (2003) observed pitch angle distributions of $>1 \mathrm{MeV}$ electrons evolving into butterfly-shaped distributions, during the recovery phase of a magnetic storm, while the electron flux was still increasing. They associated this phenomenon with acceleration of particles near $90^{\circ}$ in a localized region along the field line above or below the equator, that would then result in a broader pitch angle distribution with possibly a minimum at $90^{\circ}$ when mapped back to the equator. One of the most important types of waves that can energize electrons near $L=4$ is the whistler mode chorus via Doppler shifted cyclotron resonance (Summers et al., 1998). During active times these waves, by interaction with the $\sim \mathrm{MeV}$ electrons, may also enhance precipitation into the loss cone (Millan and Thorne, 2007) and act in this case as a loss source. These waves are generally located at the plasmapause, outside the plasmasphere in the after midnight to morning side sector, occur outside the equatorial plane and act upon electrons with energies above $100 \mathrm{keV}$. Under given conditions, chorus scattering can make a pitch angle distribution evolve into either a flat-topped or butterfly-shaped distribution (Horne et al., 2003, 2005). A similar acceleration process may act upon the electron population of a few hundred $\mathrm{keV}$ near the loss cone and slow down their decay rate.

It has been observed that the lifetimes of particles may be longer than the duration of waves and that the decay rate of waves may also tend to show continuous decrease during the storm recovery phase (Benck et al., 2008). Like the wave energy, the magnetic configuration, the plasma density along with the ion composition change with position and time. In addition, radiation belt electrons execute many drift periods within the time resolution of the flux data (in our case the time resolution for the fluxes was 1 day) used for lifetime constants determination. Yet, within one drift period, the electrons move across the different local time zones where the distribution of wave types varies. For example: a) Plasmaspheric hiss that are confined within the plasmasphere are located in the dawn to evening sector and peak in two latitude regions (Meredith et al., 2004). Their intensity is geomagnetic activity dependent. b) Whistler mode chorus waves are observed outside the plasmasphere and are located in the after midnight to dawn sector at higher latitudes. They are enhanced during active time periods. c) EMIC waves are confined to a narrow region within the plasmapause, mainly occur during geomagnetic storms and are located in the evening sector (Summers et al., 1998). d) The lightening generated whistler and VLF transmitter signals, penetrating through the ionosphere and propagating along magnetic field lines into the radiation belts are more intense on the night side than on the day side (Abel and Thorne, 1998a). It is therefore important to analyze the precipitation process and the pitch angle distribution evolution by taking into account the magnetic local time (MLT). A careful analysis of particle pitch angle distributions in MLT has been achieved by Gannon et al. (2007) and they observed a strong variation of the distributions with MLT. This kind of investigations may be complemented by LEO measurements of precipitating particles as a function of MLT. It is also fortunate that the development of a theoretical time-dependent diffusion model is under way (Albert and Shprits, 2009). In fact introducing time-dependent variables into the diffusion equation as well as magnetic local time dependent wave spectra may shed some light on until now unexplained phenomena.

\section{Conclusion}

We have analyzed SAC-C and DEMETER particle data to determine low altitude energetic electron decay timescales as a function of energy (160 keV-1.36 MeV) and L-shell (1.6$5)$. The resulting timescales are compared with existing observational lifetimes deduced mainly from fluxes measured in the equatorial plane. Our main observation is as follows: While in the inner belt and in the slot region $(L<3)$ no significant difference is observed between the low and high altitude data sets (i.e. with different $\alpha_{\mathrm{eq}}$ ), in the outer belt for the least energetic electrons $(E<500 \mathrm{keV})$ the lifetimes are up to $\sim 3$ times larger for the low altitude electrons than for high altitude ones. The difference decreases with increasing energy and vanishes for energies of about $1 \mathrm{MeV}$. This observation, that low altitude electrons with $E<500 \mathrm{keV}$ survive longer than particles having their mirror points close to the equator, sounds contradictory to what might be expected. Therefore it is recommended and justified to take a moderate critical look at the comparison: the conclusion is not based on an homogeneous data set; it was reached by comparing results deduced from data acquired by different instruments at different times. Therefore, the differences in the data acquisition and analysis procedures might introduce some uncertainties on the obtained results.

Until now, the diffusion theory does not allow for pitchangle dependent decay rates; one may expect that introduction of time-dependent variables in the diffusion equation will partially explain the observed results. Moreover, taking into account variable wave type occurrence and wave intensity as a function of MLT as the particles drift around the earth, may add some new features to the prediction of particle decay rates. Analysis of the scattering rate with regard to the wave normal angle, as a function of position and particle 
energy, may also bring some explanation to the present observation.

The theoretical evaluation of electron lifetimes is rather complex due to the fact that there are a multitude of competing mechanisms that can be operative at the same time. The present results on observational electron lifetimes should help to further clarify the understanding of electron decay rates in the radiation belts as well as in the slot region.

Acknowledgements. This work has been initiated in the context of the RABEM/SEVEM project funded under PRODEX Contract No. 90179 and finalized in the framework of the TOP-Model project funded under PRODEX Contract No. 90353: the authors are grateful to the PRODEX team and to W. Verschueren at Belspo for their support. We also thank M. Parrot and J.-A. Sauvaud (CESR) for accepting us as guest investigator in the DEMETER mission of CNES. D. Boscher, S. Bourdarie as well as R. Ecoffet are acknowledged for discussions and clarifications on the SAC-C/ICARE data.

Topical Editor I. A. Daglis thanks D. Heynderickx and another anonymous referee for their help in evaluating this paper.

\section{References}

Abel, B. and Thorne, R. M.: Electron scattering loss in Earth's inner magnetosphere 1. Dominant physical processes, J. Geophys. Res., 103(A2), 2385-2396, 1998a.

Abel, B. and Thorne, R. M.: Electron scattering loss in Earth's inner magnetosphere 2. Sensitivity to model parameters, J. Geophys. Res., 103(A2), 2397-2407, 1998b.

Albert, J. M.: Pitch-angle diffusion as seen by CRRES, Adv. Space Res., 25, 2343-2346, 2000.

Albert, J. M. and Shprits, Y. Y.: Estimates of lifetimes against pitch angle diffusion, J. Atmos. Solar-Terr. Phys., 71, 16471652, doi:10.1016/j.jastp.2008.07.004, 2009.

Baker, D. N., Blake, J. B., Callis, L. B., Cummings, J. R., Hovestadt, D., Kanekal, S., Klecker, B., Mewaldt, R. A., and Zwickl, R. D.: Relativistic electron acceleration and decay time scales in the inner and outer radiation belts: SAMPEX, Geophys. Res. Lett., 21(6), 409-412, 1994.

Baker, D. N., Kanekal, S. G., Horne, R. B., Meredith, N. P., and Glauert, S. A.: Low-altitude measurements of 2-6 MeV electron trapping lifetimes at $1.5 \leq L \leq 2.5$, Geophys. Res. Lett., 34, L20110, doi:10.1029/2007GL031007, 2007.

Beall, D. S., Bostrom, C. O., and Williams, D. J.: Structure and Decay of the Starfish Radiation Belt, October 1963 to December 1965, J. Geophys. Res., 72, 3403-3424, 1967.

Benck, S., Cyamukungu, M., and Cabrera, J.: Study of correlations between waves and particle fluxes measured on board the DEMETER satellite, Adv. Space Res., 42(9), 1538-1549, doi:10.1016/j.asr.2008.03.024, 2008.

Bourdarie, S. , Boscher, D., Heynderickx, D., Stegen, K., Buehler, P., Cyamukungu, M., Stauning, P., Clucas, S., and Ecoffet, R.: Radiation Environment Research From Multiple Monitors (RERMM), Final Report, Issue 1.0, ESA/ESTEC Contract No. 16709/02/NL/EC, p. 105-116, February 2006.

Brautigam, D. and Albert, J.: Radial diffusion analysis of outer radiation belt electrons during the October 9, 1990, magnetic storm, J. Geophys. Res., 105(A1), 291-309, 2000.
Ecoffet, R., Lorfèvre, E., Corominas-murtra, A., Sicard-Piet, A., Moulin, M., Falguère, D., Nuns, T., Duzellier, S., Boscher, D., Bourdarie, S., Sauvaud, J.A., Sarrabayrouse, G., Gasiot, J., and Dusseau, L.: CNES Activities on Ionising Particle Measurements, Workshop on Ionising particle measurements in space, Noordwijk (The Netherlands), 31 January-2 February 2005.

Falguère, D., Boscher, D., Nuns, T., Duzellier, S., Bourdarie, S., Ecoffet, R., Barde, S., Cueto, J., Alonzo, C., and Hoffman, C.: In-Flight observations of the radiation environment and its effects on devices in the SAC-C polar orbit, IEEE Trans. Nuc. Sci., 49(6), 2782-2787, 2002.

Freden, S. C.: Inner-belt Van Allen radiation, Space Sci. Rev., 9(2), 198-242, 1969.

Friedel, R. H. W., Reeves, G. D., and Obara, T.: Relativistic electron dynamics in the inner magnetosphere - a review, J. Atmos. SolarTerr. Phys., 64, 265-282, 2002

Gannon, J. L., Li, X., and Heynderickx, D.: Pitch angle distribution analysis of radiation belt electrons based on Combined Release and Radiation Effects Satellite Medium Electrons A data, J. Geophys. Res., 112, A05212, doi:10.1029/2005JA011565, 2007.

Glauert, S. A. and Horne, R. B.: Calculation of pitch angle and energy diffusion coefficients with the PADIE code, J. Geophys. Res., 110, A04206, doi:10.1029/2004JA010851, 2005.

Green, J. C. and Kivelson, M. G.: Relativistic electrons in the outer radiation belt: Differentiating between acceleration mechanisms, J. Geophys. Res., 109, A03213, doi:10.1029/2003JA010153, 2004.

Horne, R. B., Meredith, N. P., Thorne, R. M., Heynderickx, D., Iles, R. H. A., and Anderson, R. R.: Evolution of energetic pitch angle distributions during storm-time electron acceleration to MeV energies, J. Geophys. Res., 108(A1), 1016, doi:10.1029/2001JA009165, 2003.

Horne, R. B., Thorne, R. M., Glauert, S. A., Albert, J. M., Meredith, N. P., and Anderson, R. R.:, Timescale for radiation belt electron acceleration by whistler mode chorus waves, J. Geophys. Res., 110, A03225, doi:10.1029/2004JA010811, 2005.

Hudson, M. K., Kress, B. T., Mueller, H.-R., Zastrow, J. A., and Blake, J. B.: Relationship of the Van Allen radiation belts to solar wind drivers, J. Atmos. Solar-Terr. Phys., 70, 708-729, 2008.

Kataoka, R. and Miyoshi, Y.: Flux enhancement of radiation belt electrons during geomagnetic storms driven by coronal mass ejections and corotating interaction regions, Space Weather, 4, S09004, doi:10.1029/2005SW000211, 2006.

Looper, M. D., Blake, J. B., Mewaldt, R. A., Cummings, J. R., and Baker, D. N.: Observations of the remnants of the ultrarelativistic electrons injected by the strong SSC of 24 March 1991, Geophys Res. Lett., 21(19), 2079-2082, 1994.

Lyons, L. R., Thorne, R. M., and Kennel, C. F.: Pitch-Angle Diffusion of Radiation Belt Electrons within the Plasmasphere, J. Geophys. Res., 77(19), 3455-3474, 1972.

McIlwain, C. E.: Processes acting upon outer zone electrons in Radiation Belts: Models and Standards, Geophysical Monograph, vol. 97, edited by: Lemaire, J. F., Heynderickx, D., and Baker, D. N., pp. 15, AGU, Washington, D.C., 1996.

Meredith, N. P., Horne, R. B., Summers, D., Thorne, R. M., Iles, R. H. A., Heynderickx, D., and Anderson, R. R.: Evidence for acceleration of outer zone electrons to relativistic energies by whistler mode chorus, Ann. Geophys., 20, 967-979, 2002, http://www.ann-geophys.net/20/967/2002/. 
Meredith, N. P., Horne, R. B., Thorne, R. M., Summers, D., and Anderson, R. R.: Substorm dependence of plasmaspheric hiss, J. Geophys. Res., 109, A06209, doi:10.1029/2004JA010387, 2004.

Meredith, N. P., Horne, R. B., Glauert, S. A., Thorne, R. M., Summers, D., Albert, J. M., and Anderson, R. R.: Energetic outer zone electron loss timescales during low geomagnetic activity, J. Geophys. Res., 111, A05212, doi:10.1029/2005JA011516, 2006.

Meredith, N. P., Horne, R. B., Glauert, S. A., and Anderson R. R.: Slot region electron loss timescales due to plasmaspheric hiss and lightning-generated whistlers, J. Geophys. Res., 112, A08214, doi:10.1029/2007JA012413, 2007.

Meredith, N. P., Horne, R. B., Glauert, S. A., Baker, D. N., Kanekal, S. G., and Albert, J. M.: Relativistic electron loss timescales in the slot region, J. Geophys. Res., 114, A03222, doi:10.1029/2008JA013889, 2009.

Millan, R. M. and Thorne, R. M.: Review of radiation belt relativistic electron losses, J. Atmos. Solar Terr. Phys., 69, 362-377, 2007.

Obara, T., Nagatsuma, T., Den, M., Miyoshi, Y., and Morioka, A., Main-phase creation of "seed" electrons in the outer radiation belt, Earth Planets Space, 52, 41-47, 2000.

O’Brien, T. P., Lorentzen, K. R., Mann, I. R., Meredith, N. P., Blake, J. B., Fennell, J. F., Looper, M. D., Milling, D. K., and Anderson, R. R.: Energization of relativistic electrons in the presence of ULF power and MeV microbursts: Evidence for dual ULF and VLF acceleration, J. Geophys. Res., 108(A8), 1329, doi:10.1029/2002JA009784, 2003.

Onsager, T. G., Chan, A. A., Fei, Y., Elkington, S. R., Green, J. C., and Singer, H. J.: The radial gradient of relativistic electrons at geosynchronous orbit, J. Geophys. Res., 109, A05221, doi:10.1029/2003JA010368, 2004

Parrot, M.: Preface - Special issue of Planetary and Space Science 'DEMETER', Planet. Space Sci., 54, 411-412, 2006.

Sauvaud, J. A., Moreau, T., Maggiolo, R., et al.: High-energy electron detection onboard DEMETER: The IDP spectrometer, description and first results on the inner belt, Planet. Space Sci., 54, 502-511, 2006.
Seki, K., Miyoshi, Y., Summers, D., and Meredith, N. P.: Comparative study of outer-zone relativistic electrons observed by Akebono and CRRES, J. Geophys. Res., 110, A02203, doi:10.1029/2004JA010655, 2005.

Shprits, Y. Y., Li, W., and Thorne, R. M.:, Controlling effect of the pitch angle scattering rates near the edge of the loss cone on electron lifetimes, J. Geophys. Res., 111, A12206, doi:10.1029/2006JA011758, 2006.

Summers, D., Thorne, R. M., and Xiao, F.: Relativistic theory of wave particle resonant diffusion with application to electron acceleration in the magnetosphere, J. Geophys. Res., 103(A9), 20487-20500, 1998.

Summers, D., Ni, B., and Meredith, N. P.: Timescales for radiation belt electron acceleration and loss due to resonant waveparticle interactions: 1. Theory, J. Geophys. Res., 112, A04206, doi:10.1029/2006JA011801, 2007a.

Summers, D., Ni, B., and Meredith, N. P.: Timescales for radiation belt electron acceleration and loss due to resonant wave-particle interactions: 2. Evaluation for VLF chorus, ELF hiss, and electromagnetic ion cyclotron waves, J. Geophys. Res., 112, A04207, doi:10.1029/2006JA011993, 2007b.

Vampola, A. L.: Natural variations in the geomagnetically trapped electron population, Proceedings of the National Symposium on Natural and Manmade Radiation in Space, 1-5 March 1971, NASA TM X-2440, edited by: Warman, E. A., Las Vegas, p. 539-547, 1971.

West Jr., H. I., Buck, R. M., and Davidson, G. T.: The Dynamics of Energetic Electrons in the Earth's Outer Radiation Belt During 1968 as Observed by the Lawrence Livermore National Laboratory's Spectrometer on Ogo 5, J. Geophys. Res., 86(A4), 2111-2142, 1981.

Williams, D. J., Arens, J. F., and Lanzerotti, L. J.: Observations of Trapped Electrons at Low and High Altitudes, J. Geophys. Res., 73(17), 5673-5696, 1968. 